\title{
Characterization of Sulfate Groups and Assessment of Anti-Coagulant Activity of Glucomannan Sulfate Prepared from Konjac Glucomannan
}

\author{
Hao Huang*, Guanghui Wang, Jun Chen and Wei Zhou \\ Biological Engineering Institute, Chemical Engineering and Technology College, Wuhan University of Science and Technology, \\ Wuhan, China \\ *For correspondence: Email: hhzy310@163.com; hhzy310@aliyun.com
}

Received: 29 December 2014

Revised accepted: 29 May 2015

\begin{abstract}
Purpose: To determine the structure of Konjac glucomannan sulfate (KOGMS) including homogeneity, sulfate group composition as well as its anti-coagulant activity in vitro.

Methods: KOGMS was prepared using chlorosulfonic acid and N, N-dicyclohexyl carbodiimide (DCC) from konjac oligo-glucomannan (KOGM). Homogeneity analysis was confirmed by cellulose acetate membrane electrophoresis. Fourier transform infrared FT-IR spectra, Laser Raman spectra and 13C Nuclear Magnetic Resonance NMR spectra were obtained and used to analyze the sulfate groups. The anti-coagulant activity of KOGM and KOGMS was evaluated in vitro using activated partial thromboplastin time (APTT), prothrombin time (PT) and thrombin time (TT) as indicators.

Results: KOGMS was shown to be a homogenous carbohydrate with a relative molecular mass ranging from $5.8 \times 10^{3}$ to $6.2 \times 10^{3} \mathrm{Da}$ and various degrees of substitution (DS) ranging from 1.28 to 1.81 . The sulfate groups were inserted at C-2, C-6 and C-3 positions of KOGM. The APTT of human platelet-poor plasma containing KOGMS was $47.2 \mathrm{~s}$ which was close to that of heparin at a concentration of 0.5 $\mathrm{mg} / \mathrm{mL}$. Furthermore, the APTT of human platelet-poor plasma containing KOGM was only $15.6 \mathrm{~s}$ which is close to that of the negative control group. For KOGMS, the values obtained in all three tests were significantly higher than those of KOGM and the negative control group $(p<0.05)$.

Conclusion: Hydroxyl groups can be substituted efficiently by sulfated groups at C-2, C-6 and C-3 positions of KOGM with little degree of degradation. In vitro anti-coagulant activity data indicates that KOGMS has a significantly stronger anti-coagulant activity than KOGM due probably to the sulfated groups in the main chain of its molecule.
\end{abstract}

Keywords: Konjac, Oligo-glucomannan sulfate, Laser Raman spectra, Anticoagulant activity

Tropical Journal of Pharmaceutical Research is indexed by Science Citation Index (SciSearch), Scopus, International Pharmaceutical Abstract, Chemical Abstracts, Embase, Index Copernicus, EBSCO, African Index Medicus, JournalSeek, Journal Citation Reports/Science Edition, Directory of Open Access Journals (DOAJ), African Journal Online, Bioline International, Open-J-Gate and Pharmacy Abstracts

\section{INTRODUCTION}

It is well known that different kinds of sulfated polysaccharides exhibit biological activity as anticoagulant, anti-thrombotic, anti-viral, antiproliferative and anti-sclerotic compounds. [1]. One of the naturally-occurring sulfated polysaccharides is heparin, a widely used anticoagulant which may increase the functional activity of platelets [2] .Heparin has some side effects including osteoporosis, thrombocytopenia and other hemorrhagic complications [3]. Furthermore, since it is a preparation obtained from animal tissues, heparin may contain spore forms of bovine encephalitis [4]. A key research priority in modern medicine is to identify and design novel anti-coagulants with reduced clinical side effects. 
Konjac is a plant of the genus Amorphophallus which has been used to isolate immunemodulating compounds and healthcare food products for a long time. The genus is native to warm sub-tropical and tropical Eastern Asia, including Japan, Southern China and Indonesia [5]. Mature konjac tubers are washed, sliced, dried and milled by Raymond mill to produce a mixed powder which is then separated to obtain konjac flour. Konjac flour contains large amounts of konjac glucomannan (KGM) at levels ranging from 51.3 to $96.9 \%$ [6]. As the main component of konjac flour, KGM is a type of multi-functional natural polymer consisting of $\beta$-D-mannose and $\beta$-D-glucose residues (molar ratio 1.6:1) linked by $\beta-1,4-g l y c o s i d i c ~ b o n d s$ which has weak biological activities such as immunity enhancement, anti-oxidation and anti-tumor properties [7].

Konjac oligo-glucomannan sulfate (KOGMS) whas been prepared from konjac oligoglucomannan (KOGM) by enzymolysis of KGM [8]. To introduce sulfate groups into KOGM, chlorosulfonic acid (CSA) was adopted with N, NDicyclohexyl carbodiimide (DCC) as a dehydration-condensation agent in the reaction system to increase the reaction rate [9]. The aim of this work was to investigate the structure and anti-coagulant activity of KOGMS.

\section{EXPERIMENTAL}

\section{Materials}

KGM was purchased from Hubei Konson Konjac Co., Ltd. (China). KOGM was obtained from Chemical Engineering and Technology College, Wuhan University of Science and Technology, Hubei Province, China. DEAE Sepharose Fast Flow and Sephadex G-10 were purchased from Shanghai Pharmacia Co., Ltd (China). All other reagents were purchased from Sigma Co., Ltd (France).

\section{Sulfated modification of KOGM}

CSA was dripped in anhydrous pyridine (PD), under agitating and cooling in ice water bath [10]. The process lasted for $20 \mathrm{~min}$ to obtain sulfated reagent. KOGM $(200 \mathrm{mg})$ was suspended in anhydrous DMF $(20 \mathrm{~mL})$ at room temperature with stirring for $30 \mathrm{~min}$, and sulfated reagent was added dropwise. Thereafter, $50 \mathrm{mg}$ DCC was added to ensure appropriate modification of polysaccharide. The mixture was stirred for $2 \mathrm{~h}$ at $50{ }^{\circ} \mathrm{C}$. Subsequently, the mixture was cooled to room temperature and $\mathrm{pH}$ adjusted to 7 - 8 using $2.5 \mathrm{~mol} / \mathrm{L} \mathrm{NaOH}$ solution. The mixture was precipitated with ethanol, dissolved in water, and dialyzed (molecular weight cutoff, 5 - $10 \mathrm{kDa}$ ) against distilled water for $48 \mathrm{~h}$ to remove pyridine, DCC, salt and potential degradation products. Four sulfated KOGMS batches (KOGMS-1 to KOGMS-4) with different DS were collected after lyophilizing.

\section{Purification of KOGMS}

The crude polysaccharide sulfate was dissolved in distilled water $(50 \mathrm{mg} / \mathrm{mL})$ and applied to a DEAE-Sepharose Fast Flow column [11]. The column was eluted with distilled water using a gradient of $\mathrm{NaCl}$ solution at a flow rate of 3 $\mathrm{ml} / \mathrm{min}$ and fractions of $2 \mathrm{ml} / \mathrm{tube}$. The polysaccharide sulfate in the eluted fractions was detected using the phenol sulfuric acid method [12]. The second positive peak eluted at 0.8 $\mathrm{mol} / \mathrm{L} \mathrm{NaCl}$ was pooled, dialyzed and lyophilized and the Sephadex G-50 was used for further purification. The fractions eluted at Kav of 0.45 were pooled and designate as KOGMS.

\section{Homogeneity analysis}

Samples (10 $\mathrm{mg} \mathrm{mL}^{-1}, 8 \mu \mathrm{L}$ ) were applied to cellulose acetate strips (Sepraphor III) [13] and electrophoresis conducted in a Gelman semimicro bath containing $\mathrm{ZnSO}_{4}$ buffer $(0.2 \mathrm{M}, \mathrm{pH}$ 5.1) for $60 \mathrm{~min}$ at $6 \mathrm{~mA}$ and $100 \mathrm{~V}$. Strips were stained with $1 \%$ Alcian Blue and destained with $5 \%$ aqueous acetic acid containing $10 \%$ ethanol. Hyaluronic acid and chondroitin sulfate were used as standard charged polysaccharides.

\section{Sulfate content and molecular mass}

Sulfur content (S, \%) of the sulfated derivatives were determined using an elemental analyzer (Vario EL, Elemental Co., Germany). DS was calculated which referred to the average number of sulfate group on each monosaccharide residue:

$D S=\{(162 \times S) /(32-102) S$

The molecular mass of KOGMS was determined by gel germeation chromatography on a TSK 3000 sw column eluted with $0.7 \% \mathrm{HAc}-\mathrm{NaAc}$ buffer solution at a flow rate of $1.0 \mathrm{~mL} / \mathrm{min}$ at 35 ${ }^{\circ} \mathrm{C}$. Elution was monitored by a refractive index detector.

\section{Sulfate group analysis}

FT-IR spectra were recorded on a Nexus FT-IR spectrophotometer (Thermo Nicolet Corporation, USA) from 4,000 to $400 \mathrm{~cm}^{-1}$. Laser Raman spectra were recorded on a Laser Raman spectrophotometer (Thermo Nicolet Corporation, 
USA) from 3,500 to $500 \mathrm{~cm}^{-1}$. 13C NMR spectra of $40 \mathrm{mg} / \mathrm{mL}$ solution in $\mathrm{D}_{2} \mathrm{O}$ was recorded at 40 ${ }^{\circ} \mathrm{C}$ with a Bruker $600 \mathrm{MHz}$ spectrometer (Germany) and chemical shifts expressed in ppm relative to the resonance of internal standard DSS.

\section{Human blood plasma presentation}

Human blood plasma was collected from healthy individual donors into conical tubes with $2.5 \%$ sodium citrate solution. The plasma was separated from blood cells by centrifuging at $5400 \mathrm{rpm}$ at $4{ }^{\circ} \mathrm{C}$ for $20 \mathrm{~min}$. The blood plasma was stored at $-70^{\circ} \mathrm{C}$ until used.

\section{Anti-coagulant activity assay in vitro}

Anti-coagulant activity was determined by activated partial thromboplastin time (APTT), prothrombin time (PT) and thrombin time (TT) coagulation assay.

APTT was used to evaluate coagulation factors including VIII, IX, XI, XII and prekallikrein in the intrinsic blood coagulation pathway. PT was used to characterize the extrinsic coagulation factors and TT was an indicator of blood coagulation status as this transforms fibrinogen into fibrin [14]. Briefly, citrated normal human plasma (90 $\mu \mathrm{L})$ was mixed with $10 \mu \mathrm{L}$ of KOGMS at different concentrations and incubated for $1 \mathrm{~min}$ at $37^{\circ} \mathrm{C}$. APTT assay reagent $(100 \mu \mathrm{L})$ was then added to the mixture and incubated for $5 \mathrm{~min}$ at $37^{\circ} \mathrm{C}$, followed by the addition of $0.05 \mathrm{mM} \mathrm{CaCl}_{2}(100$ $\mu \mathrm{L})$ and the clotting time(s) recorded using a coagulometer.

For the PT assay, citrated normal human plasma $(90 \mu \mathrm{L})$ was mixed with $10 \mu \mathrm{L}$ of a KOGMS solution and incubated for $10 \mathrm{~min}$ at $37^{\circ} \mathrm{C}$. Preincubated PT assay reagent $(200 \mu \mathrm{L})$ was then added and clotting time recorded. For the TT assay, citrated normal human plasma (190 $\mu \mathrm{L})$ was mixed with $10 \mu \mathrm{L}$ of KOGMS and incubated for $2 \mathrm{~min}$ at $37^{\circ} \mathrm{C}$. TT assay reagent $(100 \mu \mathrm{L})$ was added and the clotting time recorded. Distilled water and heparin were used as experimental controls and standards respectively. The APPT assay and other determinations were performed with at least two replicates of samples and mean values used for calculations.

\section{Statistical analysis}

Statistical significance was determined using Student's t test; differences were considered as statistically significant at $p<0.05$.

\section{RESULTS}

\section{Homogeneity of KOGMS}

Cellulose acetate electrophoresis of KOGMS (Fig. 1) showed one mobile band which appeared to indicate that KOGMS consisting of glucose and mannose was homogeneous.

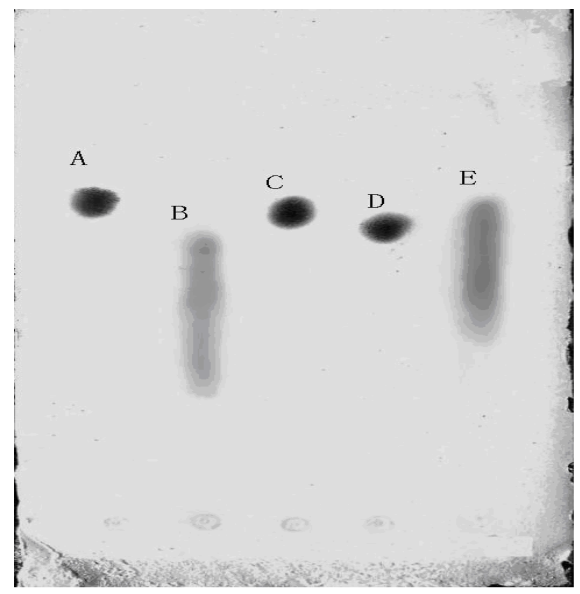

Figure 1: Cellulose acetate electrophoresis of KOGMS. (Key: A: Glucose, B: degradation of purified KOGMS, C: Mannose, D: sucrose, E: degradation of KOGM)

\section{Component analysis of KOGMS}

The molecular masses of KOGMS with different DS were investigated and the data presented in Table 1 showing the molecular masses and DS values of KOGMS increased from 5.8 to $6.2 \mathrm{kDa}$, 1.28 to 1.81 respectively. These data imply the hydroxyl groups were substituted efficiently by sulfated groups in the main chain of polysaccharide.

\section{Sulfate group analysis of KOGMS}

FT-IR spectra of native KOGM and KOGMS are shown in Figures 2 and 3. From Figure 3, the absorption peak at $3,200-3,700 \mathrm{~cm}^{-1}$ which describes an asymmetrical $\mathrm{OH}$ stretching vibration is seen to be weaker than that shown in Figure 2, indicating the $\mathrm{OH}$ groups decreased in KOGMS because of its sulfation. Compared with KOGM, two characteristic absorption peaks appeared in the FT-IR spectra of KOGMS. One at $1,224 \mathrm{~cm}^{-1}$ describing an asymmetrical $\mathrm{S}=\mathrm{O}$ stretching vibration and the other at $808 \mathrm{~cm}^{-1}$ representing a symmetrical $\mathrm{C}-\mathrm{O}-\mathrm{S}$ vibration, indicating that KOGMS may successfully sulfate. 
Table 1: Component analysis of KOGMS

\begin{tabular}{lccccccc}
\hline Sample & & & & \multicolumn{3}{c}{ Elemental analysis (\%) } & DS \\
\cline { 5 - 7 } & CSA:PD* & Time $(\mathbf{h})$ & M (kDa) & $\boldsymbol{C}$ & $\boldsymbol{H}$ & $\boldsymbol{S}$ & 1.28 \\
KOGMS-1 & $1: 5$ & 2 & 5.80 & 21.04 & 3.95 & 14.54 & 1.48 \\
KOGMS-2 & $1: 4$ & 2 & 5.85 & 19.97 & 3.87 & 15.96 & 1.67 \\
KOGMS-3 & $1: 3$ & 2 & 6.05 & 19.06 & 3.69 & 17.08 & 1.67 \\
KOGMS-4 & $1: 2$ & 2 & 6.20 & 18.65 & 3.74 & 19.33 & 1.81 \\
\hline
\end{tabular}

*Volume ratio of chlorosulfonic acid to pyridine in sulfated reagent

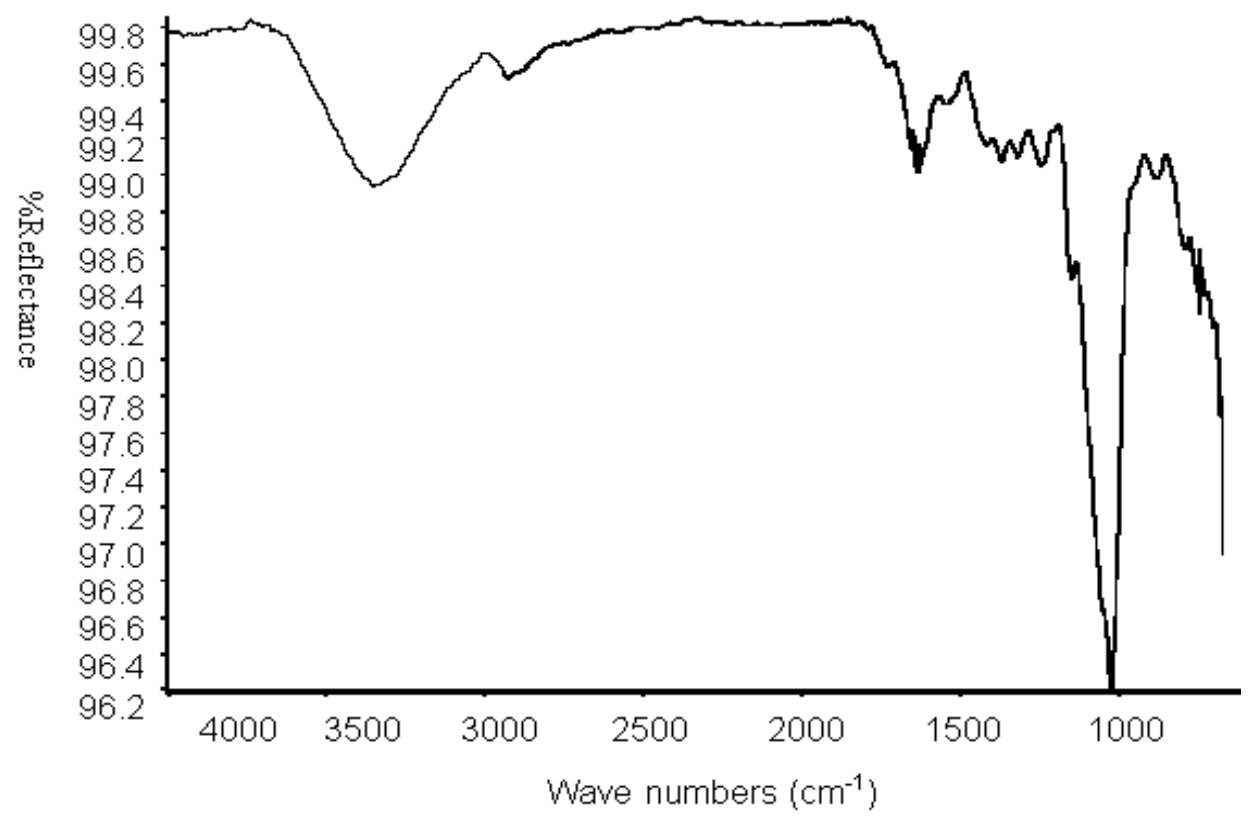

Figure 2: FT-IR spectrum of KOGM

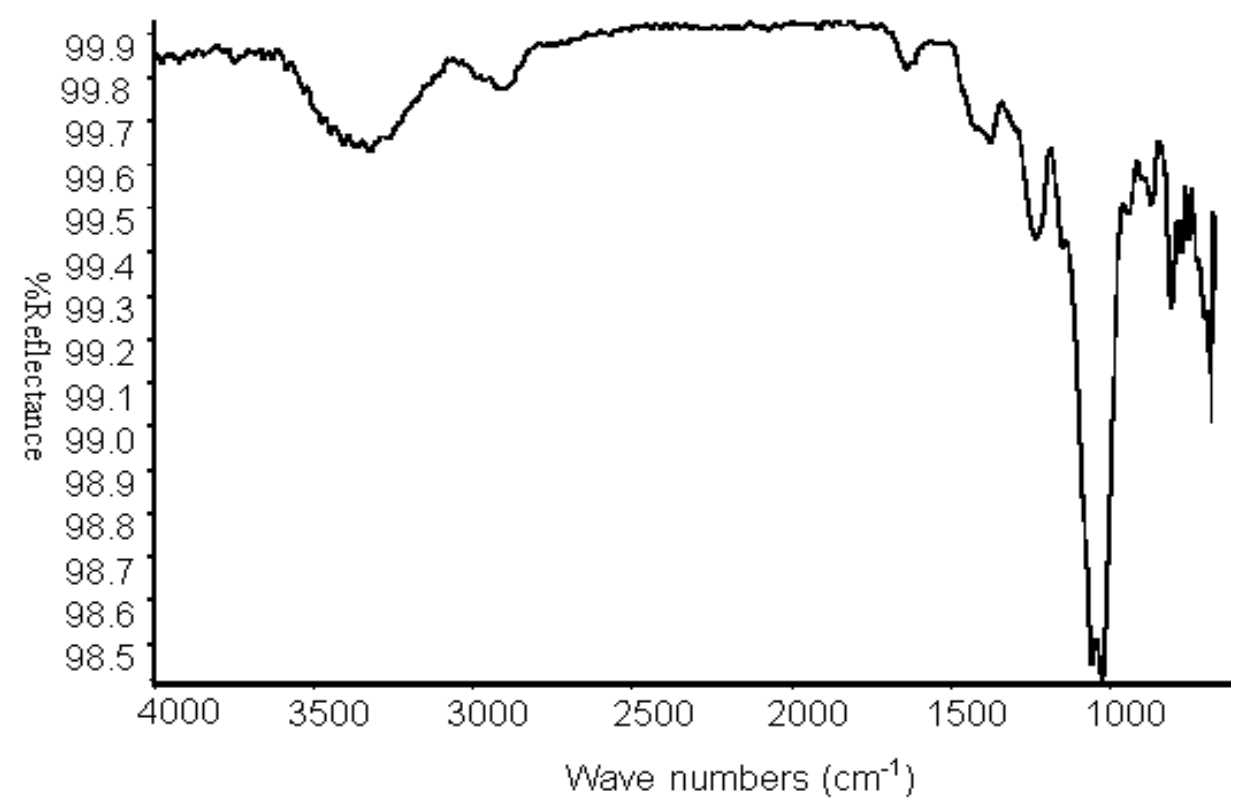

Figure 3: FT-IR spectrum of KOGMS

The Laser Raman spectra of the native KOGM and KOGMS are shown in Figures 4 and 5. From Fig. 5, CH groups decreased in KOGMS due to of its sulfation.the absorption peak had been enhanced at $1,059 \mathrm{~cm}^{-1}$ describing an asymmetrical C-O stretching vibration of cyclic ether $\mathrm{C}-\mathrm{O}-\mathrm{C}$ in the spectra of KOGMS indicating that cyclic ether increased in KOGMS due to its sulfate reaction. There were a large number of absorption peaks at $580 \mathrm{~cm}^{-1}, 447 \mathrm{~cm}^{-1}$, and 410 


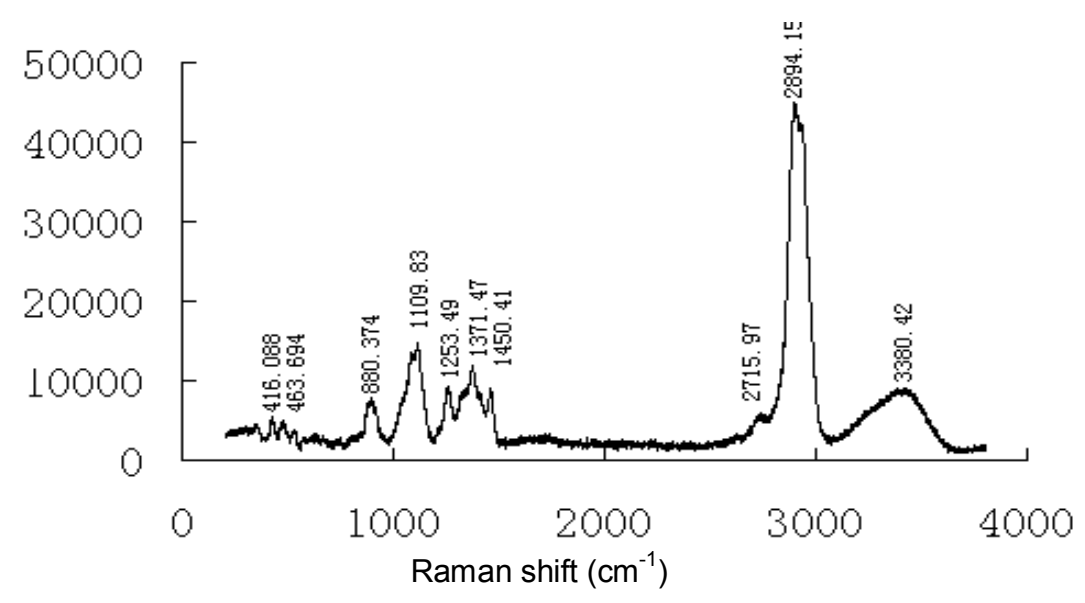

Fig 4: Laser Raman spectrum of KOGM

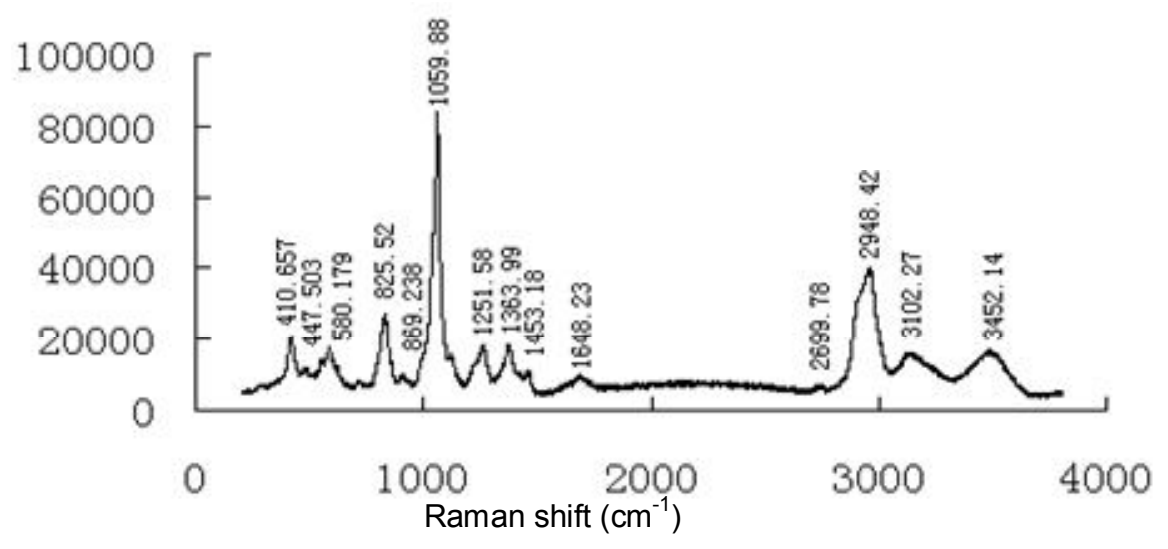

Fig 5: Laser Raman spectrum of KOGMS
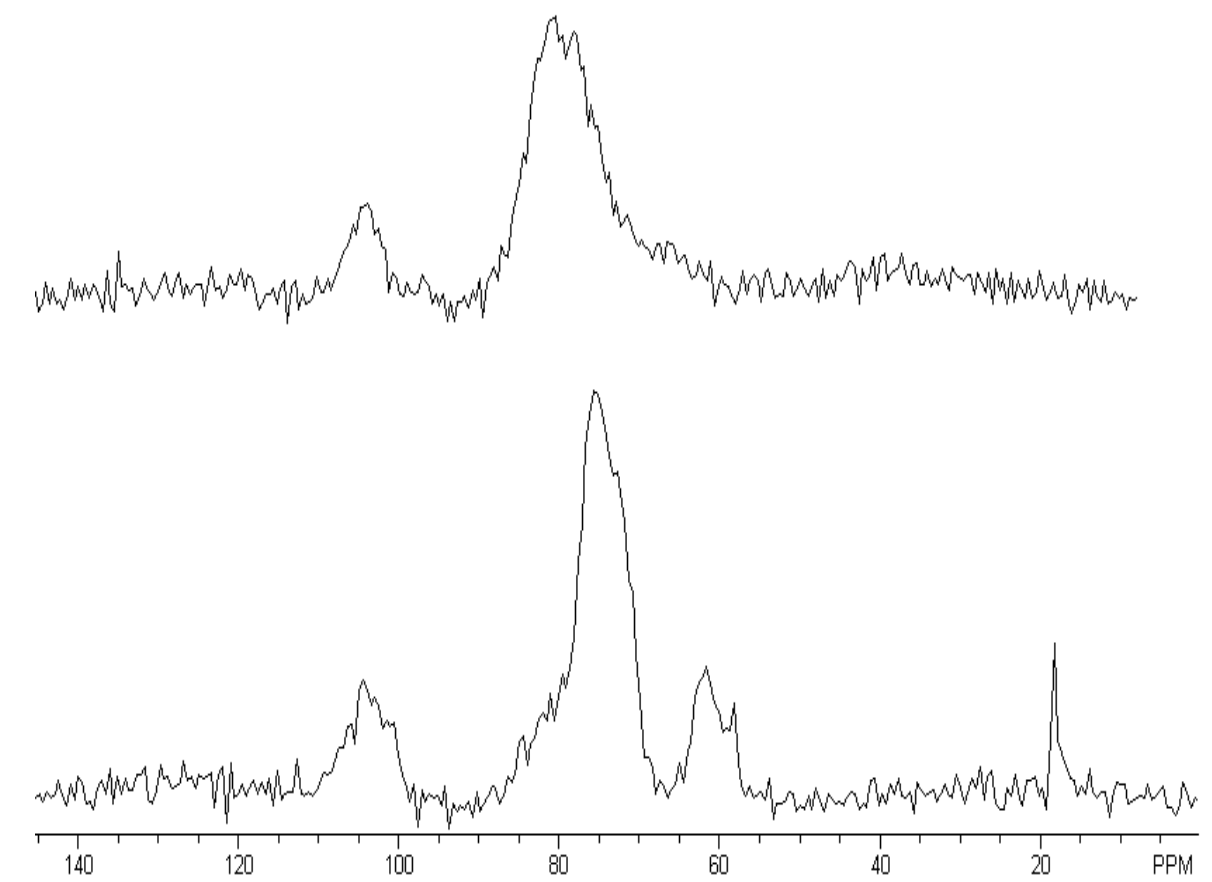

Figure 6: ${ }^{13} \mathrm{C}$ NMR spectrum of $\operatorname{KOGM}(\mathrm{a}), \mathrm{KOGMS}(\mathrm{b})(600 \mathrm{MHz})$ 
$\mathrm{cm}^{-1}$ describing a $\mathrm{CH}$ deformation vibration which could not give the definite sulfated position. At $825 \mathrm{~cm}^{-1}$, this represents a $\mathrm{CH}$ deformation vibration of mono-substitution indicating that KOGMS was successfully sulfated in $-\mathrm{OSO}_{3}$ combined with FT-IR analysis.

The sulfate position on the polysaccharide was determined by ${ }^{13} \mathrm{C}$ NMR spectrum. The ${ }^{13} \mathrm{C}$ NMR spectra for the native KOGM and KOGMS are shown in Figure 6. The ${ }^{13} \mathrm{C}$ NMR spectrum of the native KOGM exhibited six signals around 96.4, $78.2,74.9,73.1,70.9$, and $60.8 \mathrm{ppm}$, attributed to $\mathrm{C}-1, \mathrm{C}-3, \mathrm{C}-5, \mathrm{C}-2, \mathrm{C}-4$, and C-6, respectively. In comparison, there are several new signals caused by sulfate groups in KOGMS. The peak at 66.8 was assigned to the signal of $\mathrm{C}-6 \mathrm{~s}$, the peak at 72.7 was assigned to the signal of C-2s. The peak at 61.6 was weakened which indicated that C-6 had been substituted by the sulfate group.

As their strong peaks existed in the NMR spectra, the peak at 72.7 for $\mathrm{C}-2 \mathrm{~s}$ was much weaker than that of $\mathrm{C}-6 \mathrm{~s}$ at 66.8 . We can therefore conclude that the C-6 position was more active than the $\mathrm{C}-2$ position due to the steric hindrance. Furthermore, a new peak appeared at 93.9 and the native $\mathrm{C}-1$ peak at 96.4 became weaker in NMR spectra of KOGMS. New peaks at $65-75$ ppm resulted in sulfating of other positions in addition to the C-6 and C-2 positions.

\section{In vitro anti-coagulant activities}

As shown in Table 2, APTT could reach $58.6 \mathrm{~s}$ at concentration of $\mathrm{KOGMS}$ about $2 \mathrm{mg} / \mathrm{ml}$ which was close to that of heparin and three times higher than that of KOGM (16.9 s). Also as shown in Tables 3 and 4, KOGMS increased prothrombin time and thrombin time. Interestingly, preparations exhibiting higher activity in the APTT test were also more active in the two later tests. However, detection of their inhibitory activity required higher concentrations.

\section{DISCUSSION}

We have successfully introduced the sulfate process as a technique for the conversion of KOGM into a potential therapeutic product that could be further characterized and developed as an anticoagulant from KGM. Characterization of the anti-coagulant activities in vitro indicated that sulfated derivatives showed much stronger ability than native polysaccharide. These phenomena indicated much stronger anti-coagulant ability of KOGMS than that of KOGM which might be attributed to a high property of sulfate group in KOGMS.

After sulfated modification, the molecular mass of polysaccharide had not been decreased

Table 2: APTT of human platelet-poor plasma containing KOGM, KOGMS and heparin

\begin{tabular}{lccc}
\hline \multirow{2}{*}{ Sample } & \multicolumn{3}{c}{ APTT(s) at different concentrations $(\mathbf{m g} / \mathbf{m L})$} \\
\cline { 2 - 4 } & $\mathbf{0 . 5}$ & $\mathbf{1 . 0}$ & $\mathbf{2 . 0}$ \\
\hline Negative control & $14.3 \pm 0.6$ & - & - \\
KOGM & $15.6 \pm 0.3$ & $15.8 \pm 0.4$ & $16.9 \pm 0.8$ \\
KOGMS & $47.2 \pm 0.8$ & $55.3 \pm 1.6$ & $58.6 \pm 1.8$ \\
Heparin & $60.6 \pm 1.3$ & $68.4 \pm 1.5$ & - \\
\hline
\end{tabular}

Table 3: PT of human platelet-poor plasma containing KOGM, KOGMS and heparin

\begin{tabular}{lccc}
\hline \multirow{2}{*}{ Sample } & \multicolumn{3}{c}{$\mathbf{P T}(\mathbf{s})$ at different concentrations $\mathbf{( m g / m L})$} \\
\cline { 2 - 4 } & $\mathbf{0 . 5}$ & $\mathbf{1 . 0}$ & $\mathbf{2 . 0}$ \\
\hline Negative control & $11.8 \pm 1.6$ & - & - \\
KOGM & $12.3 \pm 0.5$ & $13.8 \pm 0.6$ & $15.2 \pm 1.4$ \\
KOGMS & $15.8 \pm 1.5$ & $17.9 \pm 1.6$ & $19.3 \pm 1.2$ \\
Heparin & $19.8 \pm 1.4$ & $21.2 \pm 1.8$ & - \\
\hline
\end{tabular}

Table 4: TT of human platelet-poor plasma containing KOGM, KOGMS and heparin

\begin{tabular}{lccc}
\hline \multirow{2}{*}{ Sample } & \multicolumn{3}{c}{ TT(s) at different concentrations $(\mathbf{m g} / \mathbf{m L})$} \\
\cline { 2 - 4 } & $\mathbf{0 . 5}$ & $\mathbf{1 . 0}$ & $\mathbf{2 . 0}$ \\
\hline Negative control & $6.5 \pm 1.6$ & - & - \\
KOGM & $7.6 \pm 0.9$ & $8.4 \pm 0.8$ & $8.8 \pm 0.6$ \\
KOGMS & $25.8 \pm 1.3$ & $28.3 \pm 1.6$ & $32.2 \pm 1.7$ \\
Heparin & $44.3 \pm 1.5$ & $49.3 \pm 1.8$ & - \\
\hline
\end{tabular}

Note: All the data are mean \pm SEM, $n=3$; - indicates that values were not measured 
obviously. It might be the reason of adding DCC in synthesis reaction, and making the reaction achieve dynamic equilibrium fast, which decreased the depolymerization of polysaccharide. DCC worked as a dehydration agent to afford dicyclohexylurea in the reaction.

The sulfated position on the polysaccharide was usually determined by ${ }^{13} \mathrm{C}$ NMR spectrum. It is known that the signal of $\mathrm{C}-1$ splits if the hydroxyl group on $\mathrm{C}-2$ was functionalized. It could be explained by the fact that $\mathrm{C}-2$ had been substituted which could influence the adjacent C1 to split into two peaks. As a consequence of a heterogeneous reaction, sulfated groups were distributed unevenly.

To investigate the anticoagulant properties of the sulfated polysaccharides, APTT was measured using normal human plasma. The results show that APTT is powerfully prolonged by KOGMS at $0.5 \mathrm{mgmL}^{-1}$. Usually, prolongation of APTT indicates inhibition of the intrinsic or common pathway [15]. In the present study, KOGMS inhibited the intrinsic or common pathways of coagulation.

Although lower than heparin, KOGMS exhibited much stronger anti-coagulant activity than KOGM, with increasing concentration in tests which differed significantly from the negative control and KOGM groups. APTT、PT、TT data indicate that KOGMS may possess anticoagulant activity correlating with the extrinsic coagulation process. However, KOGM had little anti-coagulant activity as per APTT, PT and TT data. The results suggest that the difference in the potency of thrombin inhibition may be due to the sugar residue, sulfation content and their structural features.

The relationship between structure and anticoagulant activity has been investigated in detail for galactan and fucans [16]. These observations show eight different sulfated polysaccharides isolated from Chlorophyta exhibit thrombin inhibition through a heparin cofactor II -dependent pathway, and their effects on the inhibition of thrombin are more potent than those of heparin or dermatan sulfate. Melo et al [17] also report the paradigm of heparinantithrombin interaction cannot be extended to other sulfated polysaccharides, and each type of polysaccharide may form a particular complex with the plasma inhibitor and the target protease. The investigations suggest that the structural requirements for the interaction of sulfated galactans with coagulation inhibitors and their target proteases are not merely a consequence of their charge density, and the structural basis of this interaction is complex, and depends on the distribution of sulfate groups and on monosaccharides composition [18]. Further structure characteristic of the sulfated polysaccharides isolated from $U$. conglobata will aid understanding of the mechanism of heparin cofactor II activation by the sulfated polysaccharides, and may ultimately lead to the development of novel antithrombotic agents.

In previous studies, a polysaccharide of fucosylated chondroitin sulfate (FucCS) isolated from sea cucumber was reported to be a potent inhibitor of $\mathrm{P}$ - and L-selectin which bound to immobilized cells [19]. Functional inhibition was also exhibited in a concentration dependent manner. Interestingly, removal of the sulfated fucose branches on the FucCS abolished the inhibitory effects both in vitro and in vivo. Our results were consistence with these findings which further confirmed sulfate group to play an important role in anti-coagulant biological activity [20].

\section{CONCLUSION}

The findings of this study indicate that hydroxyl groups are substituted efficiently by sulfate groups on the polysaccharide with a little degradation. KOGMS exhibits much stronger anti-coagulant activity than KOGM and rises with increase in APTT concentration, suggesting that KOGMS may express anti-coagulant activity that correlates with extrinsic coagulation process. The mild anti-coagulant activity of these compounds suggests they may be useful for therapeutic purposes, particularly as potential anti-thrombotic drugs.

\section{ACKNOWLEDGEMENT}

This study was supported by research grants from the International Cooperation between Poland and China, and Hubei Province National Science Fund. The authors thank Chen Xinwen, Liang Changyong, $\mathrm{Fu}$ Yourong and $\mathrm{Dr}$ Yao Xiangjie for their help.

\section{REFERENCES}

1. Hayashi K, Hayashi T, Kojima I. A natural sulfated polysaccharide, calcium spirulan, isolated from Spirulina platensis: in vitro and ex vivo evaluation of antiherpes simplex virus and anti-human immunodeficiency virus activities. AIDS Res Hum Retrov 1996; 12: 1463-1471.

2. Santos JC, Mesquita JMF, Belmiro CLR. Isolation and characterization of a heparin with low antithrombin 
activity from the body of Styela plicata (ChordataTunicata). Distinct effects on venous and arterial models of thrombosis. Thromb Res 2007; 121: 213223.

3. Mansour MB, Majdoub H, Bataille I. Polysaccharides from the skin of ray Raja radula. Partial characterization and anticoagulant activity. Thromb Res 2009; 123: 671-678.

4. Pereira MS, Benevides NMB, Melo MRS, Valente AP, Melo FR, Mourão PAS. Structure and anticoagulant activity of sulfated galactan from the red alga, Gelidium crinale. Is there a specific structural requirement for the anticoagulant action? Carbohyd Res 2005; 340: 2015-2023.

5. Katsuraya K, Okuyama K, Hatanaka K, Oshima K, Sato T, Matsuzaki K. Constitution of konjac glucomannan: chemical analysis and 13C NMR spectroscopy. Carb Polym 2003; 53: 183-189.

6. Farias WRL, Valente $A P$, Pereira MS, Mourão PAS. Structure and anticoagulant activity of sulfated galactans. J Biol Chem 2000; 275: 29299-29307.

7. Rechter S, Konig T, Auerochs S. Antiviral activity of Arthrospira-derived spirulan-like substances. Antivir Res 2006; 72: 197-206.

8. Kaji $T$, Fujiwara Y, Inomata Y. Repair of wounded monolayers of cultured bovine aortic endothelial cells is inhibited by calcium spirulan, a novel sulfated polysaccharide isolated from Spirulina platensis. Life Sci 2002; 70: 1841-1848.

9. Hayakawa $Y$, Hirashima $Y$, Yamamoto H. Mechanism of activation of heparin cofactor II by calcium spirulan. Arch Biochem Biophys 2003; 416: 47-52.

10. M'sakni NH, Majdoub H, Roudesli MS. Composition, structure and solution properties of polysaccharides extracted from leaves of Mesembryanthenum crystallinum. Eur Polym J 2006; 42: 786-795.

11. Case SE, Hamann DD. Fracture properties of konjac mannan gel: effect of gel. Food Hydrocoll 1994; 28. 147-154.
12. Wang SC, Bligh SWA, Shi SS. Structural features and anti-HIV-1 activity of novel polysaccharides from red algae Grateloupia longifolia and Grateloupia filicina. Int J Biol Macromol 2007; 41: 369-375.

13. Garnjanagoonchorn W, Wongekalak L, Engkagul $A$. Determination of chondroitin sulfate from different sources of cartilage. Chem Eng Proc 2006; 46: 465471.

14. Lu Y, Wang BL. The research progress of antitumorous effectiveness of Stichopus japonicus acid mucopolysaccharide in north of China. Am J Med Sci 2009; 337: 195-198.

15. Pereira MS, Vilela-Silva AC, Valente AP, Mourão PAS. A 2-sulfated, 3-linked-Lgalactan is an anticoagulant polysaccharide. Carbohyd Res 2002; 337: 22312238.

16. Wang $Y, Y u$ Y, Mao J. Carboxymethylated beta-glucan derived from Poria cocos with biological activities. $J$ Agr Food Chem 2009; 57: 10913-10915.

17. Lee JB, Hayashi T, Hayashi K. Further purification and structural analysis of calcium spirulan from Spirulina platensis. J Nat Prod 1998; 61: 101-104.

18. Lee JB, Srisomporn P, Hayashi K, Tanaka T, Sankawa $U$, Hayashi T. Effect of structural modification of calcium spirulan, a sulfated polysaccharide from Spirulina platensis, on antiviral activity. Chem Pharm Bull 2001; 49: 108-110.

19. Su JY, Jia SR, Chen XF, Yu HF. Morphology, cell growth, and polysaccharide production of $N$. flagelliforme in liquid suspension culture at different agitation rates. Appl Phycol 2008; 20: 213-217.

20. Wang Q, Huang X, Nakamura A, Burchard W, Hallett FR. Molecular characterization of soybean polysaccharides: An approach by size exclusion chromatography, dynamic and static light scattering methods. Carbohyd Res 2005; 340: 2637-2644. 